\title{
AEROSOL LIDAR FOR THE RELATIVE BACKSCATTER AMPLIFICATION MEASUREMENTS
}

\author{
Igor A. Razenkov*, Victor A. Banakh, Alexander I. Nadeev \\ V.E. Zuev Institute of Atmospheric Optics, Tomsk, 634055, RUSSIA, *Email: lidaroff@iao.ru
}

\begin{abstract}
Backscatter amplification presents only in a turbulent atmosphere, when the laser beam is propagates twice through the same inhomogeneities. We proposed technical solution to detect backscatter amplification. An aerosol micro pulse lidar with a beam expansion via receiving telescope was built to study this effect. Our system allows simultaneous detection of two returns from the same scattering volume: exactly on the axis of the laser beam and off the axis.
\end{abstract}

\section{INTRODUCTION}

This amplification effect arises because of the correlation of forward and backward light waves propagating through the same inhomogeneities of a random medium [1,2]. The intensity of the scattered light on the laser beam axis should be bigger than around the laser beam. Moreover, the effect of amplification is possible in a very small angle (Fresnel zone) around the optical axis of the beam. There is one approach on the measurement backscatter amplification known [3].

We designed a lidar transceiver to measure the relative backscatter amplification (RBSA). It measures the average intensity of the scattered radiation exactly on the laser beam axis and the average intensity off axis, where is no amplification at all. Our design is based on laserradar transceiver with the beam expansion via receiving telescope [4]. The same telescope works to transmit laser pulse to the atmosphere and to receive radiation scattered from molecules and aerosol particles. Such systems have good thermomechanical stability and this is important for longterm continuous observations. It is also important to get returns on-axis and off-axis at the same time. That is why our system has one transmitter channel and two receiver channels. The schematic of a lidar transceiver is shown in Fig. 1.

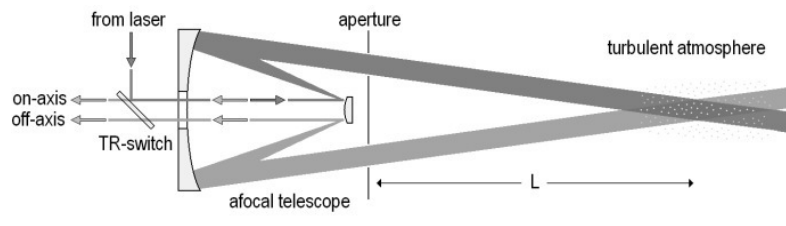

Fig. 1. Schematic drawing of the transceiver.

The main element of the system is an afocal mirror telescope. Antenna switch sends a narrow laser beam to the edge of the telescope, which is pre-focused at a distance of $(\mathrm{L}=1 \mathrm{~km})$. A screen with two round apertures is installed in front of the telescope. The laser pulse is emitted into the atmosphere through the top aperture. Scattered radiation from the atmosphere passes through both apertures to the antenna switch (transmit-receiveswitch). The first on-axis beam returned to the system in exactly the same path the laser beam as the transmitted to the atmosphere beam. The second off-axis beam from the scattering volume returns through a different path. In turbulent atmosphere in the presence of RBSA on-axial return exceeds the off-axis return. In case of a weak turbulence or absence of turbulence on-axis return will be close or equal to the off-axis return.

Equations for on-axis and off-axis lidar returns are

$$
\begin{aligned}
& P_{1}(z)=E_{0} C_{1} G_{1}(z) N(z) \beta_{\pi}(z) \frac{1}{z^{2}} T^{2}(z), \\
& P_{2}(z)=E_{0} C_{2} G_{2}(z) \beta_{\pi}(z) \frac{1}{z^{2}} T^{2}(z),
\end{aligned}
$$

where $z$ is distance; $E_{0}$ is the pulse energy; $C_{l}$ and $C_{2}$ are hardware constants includes channel transmittance, receiving aperture, and detector efficiency; $G_{l}(z)$ and $G_{2}(z)$ - geometric factors of the receiving channels; $\beta_{\pi}(z)$ is the backscatter coefficient; $N(z)$ is the backscatter amplification coefficient; $T^{2}(z)$ is the double atmospheric transmittance.

The ratio of the lidar returns $P_{1}(z)$ and $P_{2}(z)$ is proportional to the RBSA 


$$
R(z)=\frac{P_{1}(z)}{P_{2}(z)}=\frac{C_{1} G_{1}(z)}{C_{2} G_{2}(z)} \times N(z) .
$$

According to the theory [1], the amplification coefficient must be $1 \leq N(z) \leq 2$.

\section{EXPERIMENTAL SETUP}

Figure 2 shows an optical diagram of the lidar. A short light pulse from the laser 1 is directed to beam splitter $3(50 / 50)$ by turning mirror 2 . We use the fiber laser $(\lambda=532 \mathrm{~nm})$ with outgoing beam about $5 \mathrm{~mm}$ in diameter. Box 5 is energy monitor. Turning mirrors 6 and 7 direct the beam with a small offset from the axis of the Mersen telescope (mirrors 8 and 9). After the telescope, laser beam with a diameter of $45 \mathrm{~mm}$ is transmitted into the atmosphere through the top aperture of screen 10 .

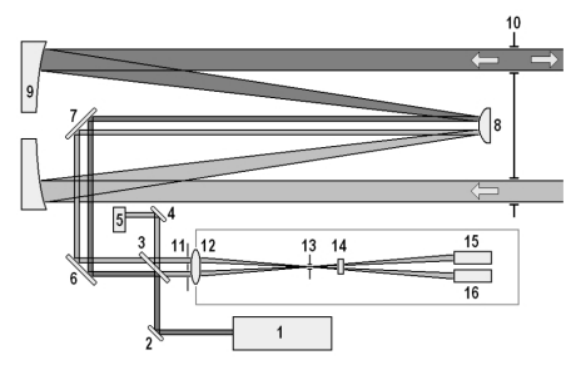

Fig. 2. Optical diagram of the RBSA lidar.

Scattered by the atmosphere radiation can be detected by the lidar only when it passes through the top and bottom holes of the screen 10. Note that the apertures' size is about equal to the Fresnel zone $(\sqrt{\lambda L})$, and the separation between them is 5 times bigger than the aperture size. Incoming beams inside the system are parallel to each other. They pass through the beam splitter 3 and the screen 11, which blocks the parasitic internally scattered radiation within the system at the moment when laser transmits the pulse.

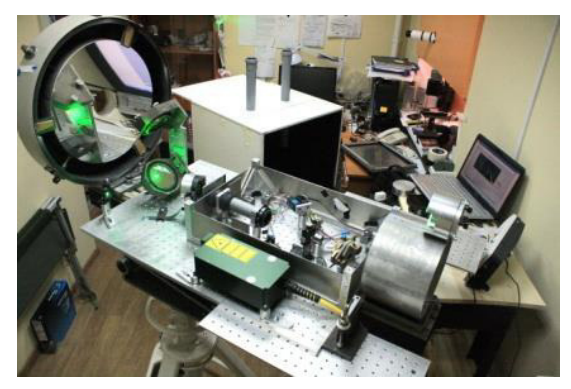

Fig. 3. Picture of the RBSA-lidar. A white cover with two in/out ports is behind the lidar.
Lens 12 focuses the beams on the field stop 13 . Size of the aperture 13, focus of lens 12 and telescope magnification $\left(10^{\times}\right)$define the field-ofview of the lidar. An interference filter 14 is installed after the field stop 13 to block the background light. Then, photodetectors 15 and 16 detect on-axis and off-axis beams respectively. The photomultiplier tubes Hamamatsu with 8-mm size of the photocathode are used. Figure 3 shows the picture of our instrument. The system parameters are listed in Table 1.

Table 1: Lidar parameters.

\begin{tabular}{|l|l|}
\hline \multicolumn{2}{|c|}{ Transmitter } \\
\hline Laser & $\begin{array}{l}\text { IPG Photonics Corp. } \\
\text { model GLPM-10-Y13 }\end{array}$ \\
\hline Wavelength & $532 \mathrm{~nm}$ \\
\hline Pulse duration & $2 \mathrm{~ns}$ \\
\hline Pulse repetition & $50 \mathrm{kHz}$ \\
\hline Laser power & $500 \mathrm{~mW}$ \\
\hline Beam quality & TEM $_{00}, \mathrm{M}^{2}<1.3$ \\
\hline Beam size & $45 \mathrm{~mm}$ \\
\hline \multicolumn{2}{|c|}{ Receiver } \\
\hline Afocal telescope & Mersen $\left(10^{\times}\right)$ \\
\hline Telescope diameter & $400 \mathrm{~mm}$ \\
\hline Channels number & 2 \\
\hline Receiving apertures & $45 \mathrm{~mm}$ \\
\hline Apertures separation & $250 \mathrm{~mm}$ \\
\hline Field-of-view & $330 \mu \mathrm{rad}$ \\
\hline Space resolution & $2 \mathrm{~m}$ \\
\hline Time resolution & $10 \mathrm{~min}$ \\
\hline Detector (PMT) & Hamamatsu H10721P \\
\hline PMT's efficiency & $10 \%$ \\
\hline
\end{tabular}

\section{CALIBRATION}

In equation (3) for the ratio $R(z)$ has hardware constants $C_{1}, C_{2}$, and geometric functions $G_{l}(z)$, $G_{2}(z)$. Let's introduce the ratio

$$
R_{0}(z)=\frac{C_{1} G_{1}(z)}{C_{2} G_{2}(z)}
$$

which is equal to $R(z)$ for $N(z) \equiv 1$. The ratio $R_{0}(z)$ is the calibration ratio, because it determines the relative sensitivity of on-axis and off-axis channels. Ratio $R_{0}(z)$ can be determined in a clean atmosphere with a weak wind, when the turbulence intensity is very low. Often these conditions are at dawn and at sunset. If to divide 
the ratio $R(z)$ (3) on the calibration ratio $R_{0}(z)$ (4) the normalized ratio $R^{\prime}(z)$ can be obtained

$$
R^{\prime}(z)=\frac{R(z)}{R_{0}(z)}=N(z) .
$$

Ratio $R^{\prime}(z)$ is equal to the relative backscatter amplification coefficient $N(z)$.

In reality, recorded returns $\tilde{P}_{1}(z)$ and $\tilde{P}_{2}(z)$ differ from the signals in the expressions (1) and (2)

$\tilde{P}_{1}(z)=P_{1}(z)+P_{B G 1}+P_{A P 1}(z)$,

$\tilde{P}_{2}(z)=P_{2}(z)+P_{B G 2}+P_{A P 2}(z)$,

where $P_{B G 1}$ and $P_{B G 2}$ - background noise, $P_{A P 1}(z)$ and $P_{A P 2}(z)$ - aftereffect (afterpulse) of photodetectors, which is caused by "blinding" of the detectors at the time of sending the laser pulse into the atmosphere and subsequent relaxation.

To obtain "clean" signals $P_{1}(z)$ and $P_{2}(z)$ is necessary to determine the response of the photodetectors $P_{A P 1}(z)$ and $P_{A P 2}(z)$ to the instant light at the moment of transmitting a laser pulse to the atmosphere. It is better do this at night before observation cycle. It is necessary to record the signals $\tilde{P}_{1}(z)$ and $\tilde{P}_{2}(z)$ with closed exit port to the atmosphere. Thus, the echo and background terms can be excluded from expressions (6) and (7). Let's call this procedure a "calibration-1". We usually perform calibration- 1 at the beginning of the measurement cycle and at the end of it.

Figure 4 presents all signals in formulas (6) and (7). Signals from on-axis channel (\#1) is shown on the left (a), and from off-axis channel (\#2) is shown on the right (b). The background (BG) has the constant value. Afterpulses curves (AP) are significantly different for the two channels. This is due to much larger pulse exposure of the detector of the on-axis channel at the moment of the shot than that for the off-axis channel. First receiving channel (on-axis) is exactly opposite to the beam splitter 3 (Fig. 2), where the laser beam is directed towards the telescope. In accordance with equations (8) and (9) "net" echoes in Fig. 4 (curves $\mathrm{P}$ ) were obtained by subtraction the afterpulse (curves $\mathrm{AP}$ ) and background (BG) from the recorded signals (curves $\mathrm{P}+\mathrm{AP}+\mathrm{BG}$ ). Data were received in October 6, 2014 at 03:40 (hereinafter, the time is local). Note the values of returns on all plots in photons per shot. Total number of laser shots for every profile is $3 \times 10^{7}$.

Signals $P_{1}(z)$ and $P_{2}(z)$ are obtained from (8) and (9) by subtracting the background (BG) and PMT's afterpulse (AP). In accordance with the formula (5), in order to determine the mutual relative sensitivity of the receiving channels a calibration ratio $R_{0}(z)$ in the absence of turbulence has to be determined (calibration-2). An example of this ratio is shown in Fig. 5, which uses the signals from Fig. 4.
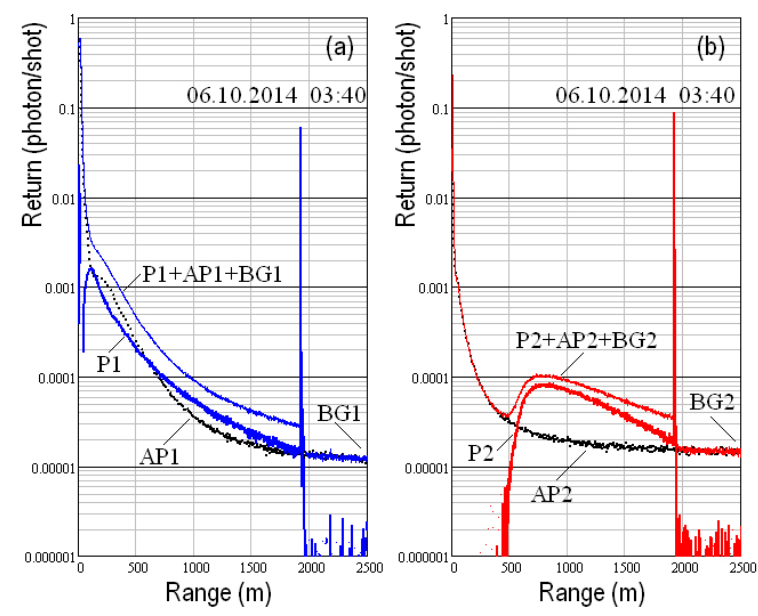

Fig. 4. Profiles of signals for (a) on-axis channel (\#1) on the left and (b) for off-axis channel (\#2) on the right. Background (BG), afterpulse (AP), return from the atmosphere $(\mathrm{P}+\mathrm{AP}+\mathrm{BG})$, and "clean" return $(\mathrm{P})$. The spike at $1950 \mathrm{~m}$ is from the solid obstacle that blocks the pulse. Data recorded on 10 October 2014 at 03:40.

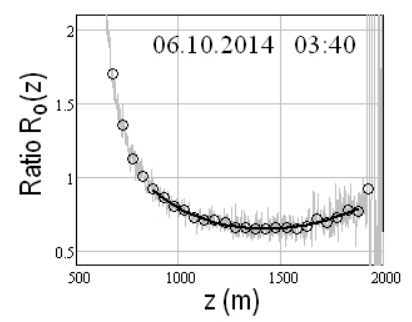

Fig. 5. The calibration ratio $R_{0}(z)$ (calibration-2) from data recorded on 10 October 2014 at 03:40 (see Fig. 3).

Our system has spatial resolution of $2 \mathrm{~m}$. A lightgray curve in Figure 5 is a ratio $R_{0}(z)$ plotted with resolution of $2 \mathrm{~m}$. To improve the statistical error signals were averaged over 25 points, and spatial resolution went down to $50 \mathrm{~m}$. In Fig. 5 
circles show $R_{0}(z)$ obtained by using the averaged signals. A smooth bold black curve in this plot is a 4th degree polynomial fit. This interpolation is used as calibration-2 to obtain the amplification coefficient $N(z)$.

\section{DATA EXAMPLES}

Ratio $R_{0}(z)$ (calibration-2) is the relative sensitivity of the receiving channels. So, $R_{0}(z)$, also can be used for comparison of returns from on-axis and off-axis channels in the analysis of experimental data. In the absence of amplification, when the ratio $N(z)=1$, far field returns $P_{1}(z)$ and $R_{0}(z) \times P_{2}(z)$ should be equal. Figure 6 shows two pairs of signals received at different time. In the data example shown in Fig. 6(a), the backscatter amplification was absent and returns are nearly equal. In case shown in Fig. 6(b) the amplification occurs, because it is clear that the on-axis return $P_{1}(z)$ is bigger than the off-axis return $R_{0}(z) \times P_{2}(z)$.
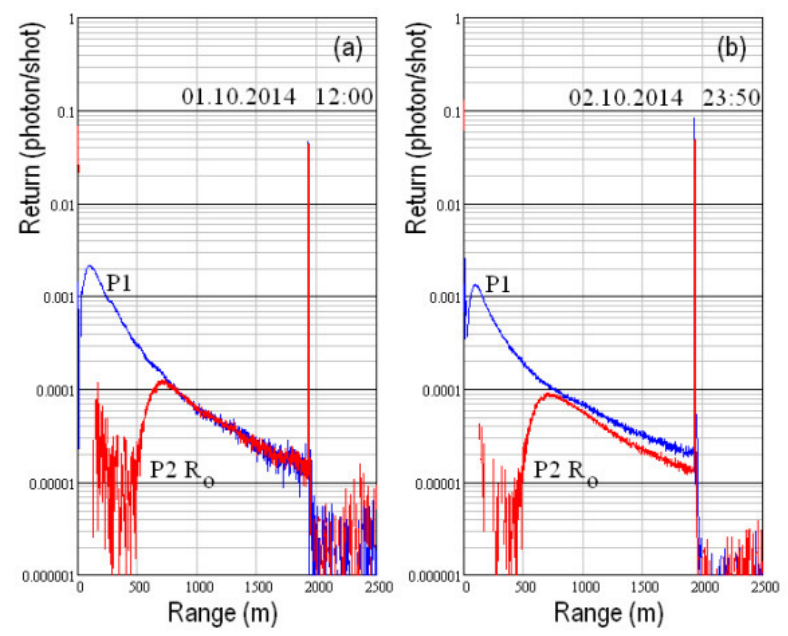

Fig. 6. On-axis (P1) and off-axis (P2) returns in (a) calm and (b) turbulent atmospheric conditions. The signal P2 is corrected on $R_{0}(z)$. Data recorded on 1 October 2014 at 12:00 and 2 October 2014 at 23:50.

Figure 7 presents the ratio $R^{\prime}(z)$ for pairs of signals described above (see Fig. 6). Calibration ratio $R_{0}(z)$ retrieved on 6 October 2014 at 03:40 (see Fig. 4 and Fig. 5) is a solid gray line. Normalized ratio $R^{\prime}(z)$ shown by circles. By dashed lines show area of $\pm 5 \%$ around $N(z)=1$, when turbulence is very low. The result of ratio $R^{\prime}(z)$ has error less than $10 \%$.
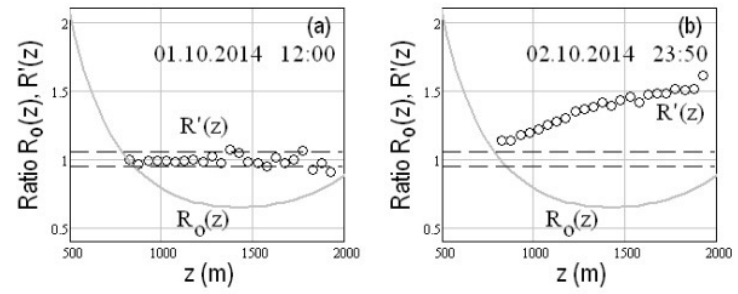

Fig. 7. Normalized ratios $R^{\prime}(z)$ obtained (a) in the absence of backscatter amplification and (b) in the presence it. Ratios (a) and (b) here are corresponds to the pairs of signals (a) and (b) in Fig. 6. Calibration ratio $R_{0}(z)$ determined from the data on 10 October 2014 at 03:40 (see Fig. 4 and Fig. 5).

Lidar is designed for long-term and unattended operation. The system currently doesn't have a remote control, but it will be implemented in the future in the newer version of the instrument. It was possible to built instrument with the laser beam expansion via receiving telescope to detect relative backscatter amplification in the atmosphere. We hope this approach is useful for future studies of the atmospheric turbulence.

\section{ACKNOWLEDGMENTS}

The authors thank Mr. Evgenii Gordeev for his technical support. This study was supported by the Russian Scientific Foundation for Maintenance and Development (Project: 14-17-00386).

\section{REFERENCES}

[1] Vinogradov A. G., Kravtsov Yu. A., and Tatarskii V. I., "The Effect of Intensification of Back Scattering by Bodies That Are Situated in a Medium Having Random Inhomogeneities," Radiophys. Quant. Electr. 16(7), 818-823 (1973).

[2] Banakh V. A. and Mironov V. L., Lidar in a Turbulent Atmosphere (Artech House, Boston and London, 1987).

[3] Gurvich A.S. // Lidar. Patent on useful model No. 116245. Registered by the State List of Useful Models of the Russian Federation of 20 may 2012 г.

[4] Razenkov, I.A., "Aerosol lidar for continuous atmospheric measurements," Atmos. Oceanic Opt. 26(1), 52-63 (2013). 